\title{
Estilos de aprendizaje de los estudiantes de medicina veterinaria de la Universidad Nacional de Rosario, Argentina
}

\author{
Laguzzi, J. ${ }^{\text {; }}$ Bernardi, S. ${ }^{2}$; Araujo, A.M. ${ }^{2}$; Ventura, A.C.3; Vigliano, F.A. ${ }^{2,4}$ \\ ${ }^{1}$ Cátedra de Producción de Bovinos de Carne, ${ }^{2}$ Cátedra de Histología I y Embriología Básica, Facultad de \\ Ciencias Veterinarias, Universidad Nacional de Rosario, Argentina; ${ }^{3}$ Instituto Rosario de \\ Investigaciones en Ciencias de la Educación -IRICE (CONICET /UNR), Argentina; \\ ${ }^{4}$ Consejo Nacional de Investigaciones Científicas y Técnicas (CONICET). \\ Correspondencia: fviglian@fveter.unr.edu.ar
}

\begin{abstract}
Resumen
Laguzzi, J.; Bernardi, S.; Araujo, A.M.; Ventura, A.C.; Vigliano, F.A.: Estilos de aprendizaje de los estudiantes de medicina veterinaria de la Universidad Nacional de Rosario, Argentina. Rev. vet. 24: 2, 151-156, 2013. El objetivo del trabajo fue identificar los estilos de aprendizaje predominantes de los estudiantes de medicina veterinaria y determinar si existen diferencias en las preferencias cognitivas entre los alumnos del ciclo de formación básica y superior de la carrera. Para ello se encuestaron 76 estudiantes de la Universidad Nacional de Rosario (Argentina), aplicando el inventario de estilos de aprendizaje de Felder y Silverman, que valora la preferencia hacia los estilos activo o reflexivo (tipo de procesamiento), sensorial o intuitivo (tipo de percepción), visual o verbal (tipo de representación) y secuencial o global (tipo de comprensión). Las preferencias cognitivas de los estudiantes encuestados de ambos ciclos de formación se orientaron hacia lo activo, sensorial, visual y secuencial. Sin embargo, en el ciclo básico se hallaron diferencias estadísticamente significativas en los estilos de procesamiento y percepción según año de ingreso. Así, los estudiantes con dos o más años de trayectoria en la carrera presentaron mayores grados de preferencia por los estilos activo y sensorial que los ingresantes. Estos resultados apoyan la hipótesis de diferencias cognitivas en el aprendizaje. En este estudio se comprobó que la mayor parte de los estudiantes analizados prefieren aprender mediante los estilos activo, sensorial, visual y secuencial. Además, existe una profundización de estas preferencias a medida que permanecen más años en la carrera. La evaluación de las características psicológicas del alumnado permitiría el diseño de clases adecuadas a sus necesidades educativas facilitando la construcción de conocimientos en la universidad.
\end{abstract}

Palabras clave: estilos de aprendizaje, medicina veterinaria, ciclo básico, ciclo superior.

\begin{abstract}
Laguzzi, J.; Bernardi, S.; Araujo, A.M.; Ventura, A.C.; Vigliano, F.A.: Learning-styles of students of veterinary medicine in the National University of Rosario, Argentina. Rev. vet. 24: 2, 151-156, 2013. The aim of this study was to identify the main learning-styles profiles of the students of veterinary medicine, as well as to determine if differences in cognitive preferences between students of basic or superior training cycle of the career exist. Seventy-six students of the School of Veterinary Sciences of the National University of Rosario (Argentina) were polled using the index of learning styles developed by Felder and Silverman. This index assess the preference toward active or reflective (processing dimension), sensing or intuitive (perception dimension), visual or verbal (representation dimension) and sequential or global (understanding dimension) styles. The cognitive preferences of polled students were orientated to active, sensing, visual and sequential styles. However, there were statistically significant differences in processing and perception dimension in the basic training cycle depending on the date of beginning of the course. Thus, students with two or more years in the career showed higher preference levels to active and sensing styles than the new students. These results support the hypothesis of cognitive differences in learning. In this study, it was confirmed that most of assessed students preferred to learn by means of active, sensing, visual and sequential styles. Moreover, these preferences were deeper as students remain more years in the career. The assessment of the psychological profile of the student
\end{abstract}


body would allow the design of suitable lessons making easier the knowledge building in the university.

Key words: learning styles, veterinary medicine, basic and superior training cycles.

\section{INTRODUCCIÓN}

El proceso de enseñanza y aprendizaje ha sido estudiado fundamentalmente en ámbitos educativos iniciales y medios. Las investigaciones sobre esos procesos en estudiantes universitarios están en aumento. En general predominan los enfoques que evalúan ciertos rasgos individuales del docente (actitudes, aptitudes, estilos de enseñanza, entre otros) suponiendo que determinan por sí mismos los logros de aprendizaje de los alumnos ${ }^{16}$.

Las investigaciones en el área de la teoría experiencial del aprendizaje demostraron que las personas aprenden de diversas formas ${ }^{9}$. Por eso es fundamental que la selección de las estrategias de enseñanza para promover el aprendizaje de los estudiantes considere sus estilos o formas típicas de aprendizaje.

Existe un consenso relativamente generalizado en definir a los estilos de aprendizaje como "las formas preferidas de un individuo de responder a tareas de aprendizaje que cambian dependiendo del medio ambiente o contexto. Por lo tanto el estilo de aprendizaje de una persona es maleable" ${ }^{15}$.

En el ámbito universitario predominan dos corrientes teóricas relacionadas con los estilos de aprendizaje y enseñanza: la línea de investigación anglosajona ${ }^{7} \mathrm{y}$ la línea de investigación española ${ }^{1}$. El presente trabajo se enmarca en la línea anglosajona dado que se dirige al estudio de aspectos cognitivos y perceptivos en conjunto y sus métodos de investigación están diseñados específicamente para la población universitaria.

El modelo de Felder y Silverman (1988) sistematizó las preferencias cognitivas involucradas en el aprendizaje mediante cuatro dimensiones: (1) percepción, (2) procesamiento, (3) representación y (4) comprensión. Cada una de estas dimensiones está constituida por dos estilos de aprendizaje opuestos: (1) sensorial-intuitivo, (2) activo-reflexivo, (3) visual-verbal y (4) secuencialglobal, respectivamente. Las características de cada estilo de aprendizaje son las siguientes ${ }^{18}$ :

- Sensorial, se orienta hacia la percepción de hechos de la realidad y detalles. Refiere a un tipo de pensamiento concreto, práctico y procedimental.

- Intuitivo, tiende hacia el descubrimiento de relaciones entre conceptos y significados subyacentes. Alude a habilidades creativas e innovadoras.

- Activo, indica preferencias hacia el procesamiento a través de la aplicación de los conocimientos y el trabajo en grupo.
- Reflexivo, señala habilidades para adquirir conocimientos mediante la escucha y el pensamiento individual sobre los contenidos.

- Visual, representa mejor el contenido a través de materiales figurativos como diagramas, gráficas, películas, demostraciones, entre otros.

- Verbal, alude a las preferencias por las explicaciones orales u escritas.

- Secuencial, se orienta hacia una comprensión de tipo analítica siguiendo procesos lineales y predeterminados.

- Global, tiende hacia el entendimiento holístico captando el sentido amplio de los contenidos. Esta característica puede conllevar dificultades para explicar el modo en que llevan a cabo la comprensión de los conceptos y conclusiones.

Recientemente, un grupo de trabajo publicó una revisión de investigaciones empíricas y teóricas acerca de los estilos y estrategias de aprendizaje de los últimos diez años ${ }^{3}$. El consenso radicaría en la relación existente entre las características de los estilos de aprendizaje y la carrera que eligen los estudiantes. Esta relación permite inferir que dependiendo del campo disciplinar, el estudiante desarrollará unos u otros estilos de aprendizaje en función de los contenidos, metodologías, información y exigencias de cada carrera.

En el área de la salud, se han investigado predominantemente los estilos de aprendizaje de los estudiantes de medicina 4, 6, 10,12 y enfermería 2, 5,20. Sin embargo, existe un menor número de antecedentes específicos en medicina veterinaria.

Al analizar los estilos de aprendizaje de estudiantes de primer año de medicina veterinaria de la Universidad Austral (Chile), se demostró que las preferencias del grupo fueron por el estilo activo y, en menor intensidad, por el estilo pragmático. Los estudiantes activos poseen un perfil animador, descubridor, improvisador, espontáneo y arriesgado, mientras que los estudiantes pragmáticos tienden a ser experimentadores, prácticos, directos, eficaces y realistas ${ }^{21}$.

En coherencia con estos resultados, fueron evaluadas las preferencias de 150 estudiantes de medicina veterinaria de la Universidad de Carolina del Norte (EEUU) de tres cohortes consecutivas ${ }^{13}$. Los resultados mostraron que el patrón predominante de los participantes fue: sensorial-activo-visual-secuencial, relevando en forma separada los siguientes valores de cada estilo: sensorial (79\%), activo (57\%), visual (77\%) y secuencial (69\%).

El objetivo general de esta investigación fue identificar los estilos de aprendizaje predominantes que 
poseen estudiantes de medicina veterinaria que cursan los ciclos básico (primer año) y superior (quinto año) en la Universidad Nacional de Rosario y determinar si existen diferencias en sus preferencias cognitivas. Considerando que una de las hipótesis principales de la teoría de los estilos de aprendizaje es que se facilita el proceso de enseñanza y aprendizaje cuando el docente enseña en el estilo predominante del estudiante ${ }^{1}$, el propósito final de este estudio es diseñar estrategias de enseñanza adecuadas para promover aprendizajes significativos y mejorar el rendimiento académico de los estudiantes.

\section{MATERIAL Y MÉTODOS}

Participantes. La muestra estuvo conformada por 76 estudiantes de la Facultad de Ciencias Veterinarias de la Universidad Nacional de Rosario (Argentina) de los cuales 46 cursaban el ciclo básico habiendo ingresado en distintos años y 30 el ciclo superior. Según el plan de estudios vigente, las asignaturas del ciclo básico se ubican en los primeros dos años de la formación académica y tienen como objetivo aportar los conocimientos sobre las bases estructurales y funcionales de los animales objeto de estudio. Por su parte, las asignaturas del ciclo superior se sitúan en los últimos años del trayecto universitario y proveen los conocimientos básicos que generan las habilidades y actitudes necesarias para dar sustento a la formación profesional, permitiendo completar y profundizar la formación teórica y práctica en las distintas áreas profesionales que comprende la medicina veterinaria ${ }^{17}$.

Materiales. El llamado index of learning styles (ILS) es un instrumento de administración colectiva ${ }^{8}$ disponible en el URL < http://www.engr.ncsu.edu/learningstyles/ilsweb.html $>$, que al ser traducido al idioma castellano recibió la denominación de inventario de estilos de aprendizaje ${ }^{18}$. Se aplicó el ILS que mide la variable "estilos de aprendizaje" operacionalizada mediante cuatro escalas o dimensiones cognitivas: (1) percepción, (2) procesamiento, (3) representación y (4) comprensión. Cada dimensión se evalúa a través de 44 preguntas dicotómicas (A ó B), las opciones "A" corresponden a las preferencias: activo, sensorial, visual y secuencial, y se le asigna una unidad negativa (-1) por ítem. Las opciones " $\mathrm{B}$ " refieren a las preferencias: reflexivo, intuitivo, verbal y global, y se le asigna una unidad positiva $(+1)$. La suma de las respuestas a las 11 preguntas proporciona el índice de cada dimensión, donde el signo sólo sitúa la orientación de la preferencia hacia uno u otro lado de la misma.

Procedimientos y análisis de datos. El diseño de la investigación fue descriptivo-correlacional de corte transversal. En forma preliminar, se llevó a cabo una prueba piloto con 263 estudiantes regulares de primer año de distintas carreras universitarias: 59 de ingeniería, 93 de licenciatura en psicopedagogía y 111 de licenciatura de kinesiología ${ }^{19}$. Esta evaluación inicial permitió realizar ajustes en relación con el formato de presentación del instrumento (adecuación y extensión de los enunciados, ordenamiento lógico de los ítems, tiempo estimado de aplicación) así como examinar el contenido de los enunciados (comprensibilidad, resistencia o rechazos sistemáticos y fatiga).

Respecto a las condiciones de la administración del instrumento, tanto en la prueba piloto así como en su aplicación definitiva, el formato de trabajo fue individual, anónimo y auto-administrado en versión papel. La consigna consistió en invitarlos a aproximarse a sus modalidades de aprendizaje, solicitando su colaboración. Luego de la entrega del material, se explicaron las instrucciones en forma oral y se ejemplificó de manera gráfica el modo de respuesta del ILS. La aplicación fue independiente para cada curso y se realizó en una clase bajo la supervisión docente.

Por otro lado, se atendieron los resguardos éticos informando a los participantes el encuadre y finalidad de la actividad propuesta así como se respetaron los protocolos de consentimiento informado. Respecto al análisis de datos, la identificación de los estilos predominantes de los estudiantes y su comparación según nivel académico se analizó mediante técnicas estadísticas descriptivas. Además se realizó una tabla de contingencia (2x3) evaluando dos estilos de aprendizaje en tres grupos de estudiantes (ciclo básico-ingreso 2012, ciclo básico-ingresos 2009, 2010 y 2011, y ciclo superior). Para calcular las diferencias entre grupos se utilizó una prueba de chi cuadrado, estableciendo diferencias significativas a $\mathrm{p}<0,05$. El procesamiento fue asistido por el programa informático SPSS (versión 17.0).

\section{RESULTADOS Y DISCUSIÓN}

Se observó una marcada tendencia en los estudiantes analizados hacia lo activo, sensorial, visual y secuencial, resultados que coinciden con los obtenidos en otros contextos universitarios en donde se imparte la carrera ${ }^{13,21}$. Al evaluar las preferencias de 150 estudiantes de tres cohortes consecutivas de una carrera de medicina veterinaria de EEUU, se observó que el patrón predominante de los participantes fue: sensorialactivo-visual-secuencial, relevando en forma separada los siguientes valores de cada estilo: sensorial (79\%), activo (57\%), visual (77\%) y secuencial $(69 \%){ }^{13}$. Tal patrón coincide con los resultados obtenidos en el presente trabajo, tanto para los estudiantes del ciclo básico como del superior, si bien los porcentajes correspondientes a cada estilo fueron más elevados en los estudiantes argentinos.

Al analizar los resultados por ciclo de la carrera, no se encontraron diferencias estadísticamente significativas en las preferencias de estilos de aprendizaje entre los estudiantes del ciclo básico y superior. En este sentido, los estudiantes preferirían aprender mediante la práctica, el trabajo cooperativo y la aplicación de los contenidos, así como tendrían mayores facilidades 
para adquirir conceptos presentados mediante materiales concretos (casos, datos, hechos reales) e ilustrados con contenidos figurativos (figuras, imágenes, tablas). Además, comprenderían más rápidamente las explicaciones y los desarrollos ordenados lógicamente desde lo particular hacia lo general.
Cabe destacar que, si bien no existieron diferencias significativas entre alumnos del ciclo básico y superior, en todas las dimensiones, a excepción de la representación, se observa una mayor preferencia hacia los estilos activo, sensorial y secuencial en los alumnos del ciclo superior (Figuras 1a-1d).

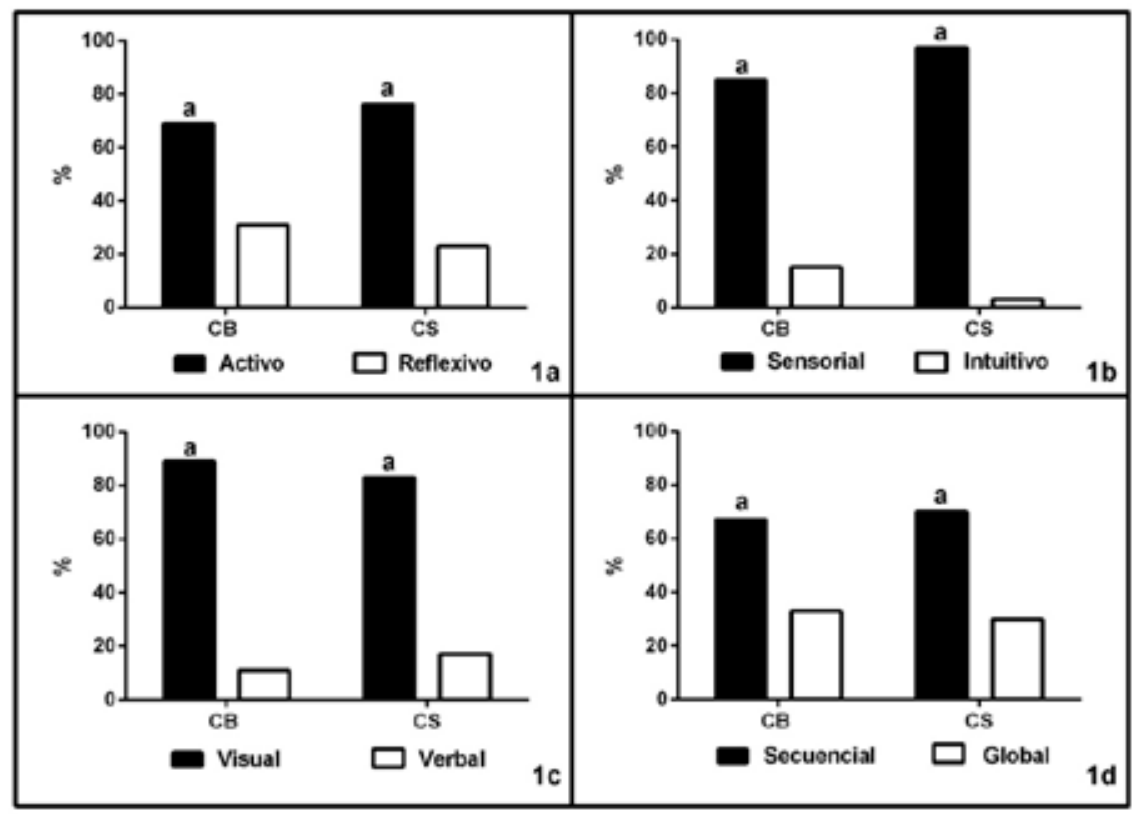

Figura 1. Estilos de aprendizaje para las dimensiones a. Procesamiento, b. Percepción, c. Representación y d. Comprensión, de los estudiantes del ciclo básico (CB) y superior (CS). Letras distintas indican diferencias significativas entre grupos.

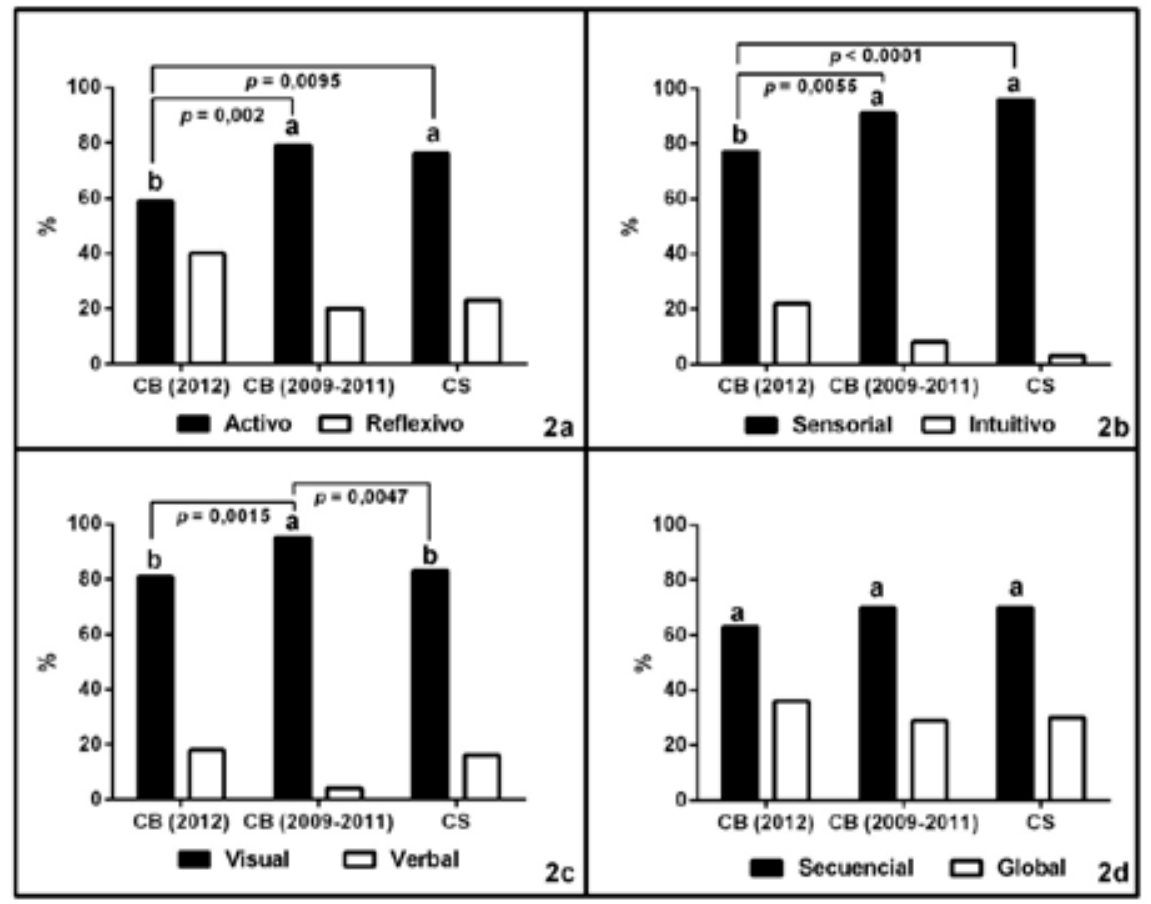

Figura 2. Estilos de aprendizaje para las dimensiones a. Procesamiento, b. Percepción, c. Representación y d. Comprensión, de los estudiantes del ciclo básico con menos de un año de permanencia en la carrera (CB 2012), más de un año de permanencia en la carrera (CB 2009-2011) y superior (CS). Letras distintas indican diferencias significativas entre grupos. 
Por un lado, los estudiantes con una mayor antigüedad en la carrera, presentaron mayores grados de preferencias hacia los estilos sensorial y activo; por otro, la representación fue una dimensión que discriminó los resultados de la muestra según el ciclo académico (básico-superior). En función de ello, es posible inferir que a medida que van desarrollando competencias en el ámbito de la propia disciplina van modificando sus preferencias, de forma que van adquiriendo más interés por la observación, los materiales figurativos, el análisis de los hechos, el trabajo metódico sistematizado así como por la experimentación, la aplicación y llevando a la práctica lo aprendido.

En base a la tendencia antes mencionada, y bajo la hipótesis de que la carrera afianza estilos de aprendizaje relacionados con las competencias profesionales del médico veterinario, se analizaron los resultados discriminando a los estudiantes del ciclo básico en alumnos de menos de un año de permanencia en la carrera (ciclo básico-ingreso 2012) y estudiantes de más de un año de permanencia en el ciclo básico (ciclo básico-ingreso 2009 a 2011). Esto permitió hallar diferencias estadísticamente significativas en las dimensiones de procesamiento y percepción entre los estudiantes que cursan por primera vez, respecto de aquellos que ya tienen dos o más años en la Facultad y los alumnos del ciclo superior (Figuras 2a-2b).

Es posible apreciar que los estudiantes que se incorporaron hace más de un año a este sistema educativo universitario (alumnos ciclo básico 2009-2011 y ciclo superior) presentaron una mayor preferencia por lo activo y lo sensorial respecto a los ingresantes 2012. Por su parte, la dimensión de representación mostró diferencias significativas hacia lo visual en los estudiantes del ciclo básico (2009-2011) respecto a los ingresantes 2012 y los alumnos del ciclo superior (Figura 2c). Por último, no se encontraron diferencias significativas entre ninguno de los tres grupos discriminados al analizar la dimensión comprensión (Figura 2d).

Apoyando los estudios que evidencian la existencia de diferencias cognitivas individuales según el tipo de comunidad académica-disciplinar de los estudiantes es posible suponer que esta transformación y tendencia hacia la consolidación de un perfil propio del médico veterinario podría deberse a dos razones que no son mutuamente excluyentes ${ }^{3}$. Por un lado, una adaptación cognitiva de los estudiantes a las formas de enseñar de los docentes y, por el otro, una adaptación a los estilos de aprendizaje relacionados con las disciplinas estudiadas en los primeros años de la carrera.

En suma, es notable la capacidad de los estudiantes para modificar su forma de aprender en función de sus estudios. Las metodologías de enseñanza y las estrategias didácticas, posiblemente encaucen las preferencias de los alumnos en un sentido. Si el estudio de la disciplina de medicina veterinaria requiere un perfil sensorial-activo-visual-secuencial o los docentes lo están potenciando con los métodos utilizados, cabe esperar que los estudiantes con mayor preferencia por estos estilos obtengan mayores logros académicos a lo largo de su formación, mientras que los alumnos con un perfil opuesto tengan mayores obstáculos para aprender y, por lo tanto, necesiten un mayor apoyo por parte del profesorado.

Este idea se basa en la hipótesis de emparejamiento o ajuste entre estilos de aprendizaje y estilos de enseñanza en entornos universitarios, una "variable proceso" que puede influir en la determinación de los logros del alumnado ${ }^{14}$. Debido a ello, es necesario buscar alternativas de enseñanza para potenciar el desarrollo de los estilos preferidos por los estudiantes: planteamientos de preguntas, elaboración de mapas o redes conceptuales, torbellino de ideas, realización de posters, debates, uso del pensamiento analógico o búsqueda de semejanzas y contrastes, debates, observación y discusión sobre imágenes, informaciones alusivas ${ }^{11}$.

A modo de conclusión, es preciso señalar que los datos de este estudio operan como tendencias preliminares de una aproximación exploratoria a la población de estudio, las cuales motivan futuras investigaciones confirmatorias. A los fines de obtener una mayor evidencia científica sería necesario llevar a cabo un estudio longitudinal de seguimiento de cohortes en el transcurso de la formación académica con el fin de analizar también si existe relación entre estilos de aprendizaje y estilos de enseñanza. Por otro lado, nuestro mayor interés es que este trabajo actúe como disparador de otros similares en las distintas unidades académicas dedicadas a la enseñanza de las ciencias veterinarias, posibilitando así conocer los estilos de aprendizaje de los estudiantes de nuestro país.

Agradecimientos. Los autores agradecen al Dr. Raúl Gagliardi por sus sugerencias luego de la lectura crítica del manuscrito.

\section{REFERENCIAS}

1. Alonso C, Gallego D, Honey P. 1999. Los estilos de aprendizaje, $2^{\circ}$ ed., Ed. Mensajero, Bilbao, p. 72-85.

2. Báez F, Hernández J, Pérez J. 2007. En Puebla, México: estilos de aprendizaje de los estudiantes de enfermería. Aquichán 7: 219-226.

3. Bahamón M, Vianchá M, Alarcón L, Bohórquez C. 2012. Estilos y estrategias de aprendizaje: una revisión empírica y conceptual de los últimos diez años. Pensamiento Psicológico 10: 1129-1144.

4. Borracci R, Guthman G, Rubio M, Arribalzaga E. 2008. Estilos de aprendizaje en estudiantes universitarios y médicos residentes. Educación Médica 11: 229-238.

5. Canalejas M, Martínez M, Pineda M, Vera M, Soto M, Martín A, Cid M. 2005. Estilos de aprendizaje en los estudiantes de enfermería. Educación Médica 8: 83-90.

6. Díaz G, Mora S, Lafuente J, Gargiulo P, Bianchi R, Terán C, Gorena D, Arce J, Escanero J. 2009. Estilos de aprendizaje de estudiantes de medicina en universidades latinoamericanas y españolas: relación con los contextos geográficos y curriculares. Educación Médica 12: 183-194. 
7. Felder R, Silverman L. 1988. Learning and teaching styles in engineering education. Engr Education 78: 674681.

8. Felder R, Soloman B. 1996. Learning styles and strategies. Disponible on line: http://www.engr.ncsu.edu/learningstyles/ilsweb.html.

9. Joy S, Kolb D. 2009. Are there cultural differences in learning style? International Journal of Intercultural Relations 33: 69-85.

10. Lima A, Bettati M, Baratta S, Falconi M, Sokn F, Galli A, Barrero C, Cagide A, Iglesias R. 2006. Learning strategies used by cardiology residents: assessment of learning styles and their correlations. Education Health: change in learning and practice 19: 289-297.

11. Martínez Geijo P. 2008. Estilos de aprendizaje: pautas metodológicas para trabajar en el aula. Revista Complutense de Educación 19: 77-94.

12. Napoli J, Formosa M, Urssi L. 2010. Evaluación comparativa de los estilos de aprendizaje en estudiantes de pregrado de patología, médicos residentes y profesionales de la carrera docente. Revista de la Asociación Médica Argentina 123: 18-22.

13. Neel JA, Grindem CB. 2010. Learning-style profiles of 150 veterinary medical students. Journal of Veterinary Medical Education 37: 347-352.

14. Pasher H, McDaniel M, Rohrer D, Bjork R. 2008. Learning styles. Concepts and evidence. Psychological Science in the Public Interest 9: 105-119.
15. Peterson E, Rayner S, Armstrong S. 2009. Researching the psychology of cognitive style and learning style: Is there really a future? Learning and Individual Differences 19: 518-523.

16. Prados M, Cubero M, de la Mata M. 2010. ¿Mediante qué estructuras interactivas se relacionan profesorado y alumnado en las aulas universitarias? Electronic Journal of Research in Educational Psychology 8: 163-194.

17. Universidad Nacional de Rosario. 2009. Plan de estudios de la carrera de Medicina Veterinaria de la Facultad de Ciencias Veterinarias (Resolución C.S. $N^{\circ}$ 588/2009). Rosario.

18. Rodríguez Suárez J. 2002. Educación médica. Aprendizaje basado en problemas, Médica Panamericana, México, p. $32-42$.

19. Ventura AC, Gagliardi R, Moscoloni N. 2012. Estudio descriptivo de los estilos de aprendizaje de estudiantes universitarios argentinos. Revista de Estilos de Aprendizaje 9: 71-84.

20. Vera Cortes M. 2010. Modificación del estilo de aprendizaje de enfermeras con la experiencia profesional. Reduca 2: 1325-1340.

21. Von Chrismar A. 2005. Identificación de los estilos de aprendizaje y propuesta de orientación pedagógica para estudiantes de la Universidad Austral de Chile. Tesis de Maestría (MSci), Valdivia, Chile. Recuperado el 29 de noviembre de 2012, http://cybertesis.uach.cl/tesis/uach/2005/ egc932i/doc/egc932i.pdf. 